\title{
Exciton Lifetime in PbS Quantum Dots in Glass
}

\author{
Peter D Persans, A. Filin, N. E. Berry, F. Huang, and E. Chan \\ Physics Department, Rensselaer Polytechnic Institute, 110 Eighth Street, Troy, NY, 12180
}

\begin{abstract}
We report experimental measurements and analysis of HOMO-LUMO transition photoluminescence and photoinduced bleaching magnitude and decay kinetics in lead sulfide quantum dots in glass. We find that the radiative lifetime is independent of temperature from $77 \mathrm{~K}$ to $500 \mathrm{~K}$. The lifetime of the HOMO-LUMO state decreases dramatically for T>300K, indicating thermally activated nonradiative recombination. The excitation decay time is much greater than the radiative lifetime and increases monotonically with decreasing temperature with decay times of $2 \mu$ s at room temperature and $4-10 \mu$ s at $77 \mathrm{~K}$.
\end{abstract}

\section{INTRODUCTION}

A single gap solar cell has an optimal unconcentrated solar energy conversion efficiency of $\sim 32 \%$ for a band gap of $\sim 1.5 \mathrm{eV}$. The discovery of efficient multiexciton generation by high energy photons in quantum dots $[2,3]$ may lead to significant improvements in efficiency with a concomitant decrease in the optimal band gap. When multiexciton generation is considered, quantum dots with their lowest excited state near $0.5-0.8 \mathrm{eV}$ are particularly interesting for solar photovoltaic applications [4]. $\mathrm{PbS}$ and $\mathrm{PbSe}$ are prototypical materials that satisfy the desired properties of narrow gap (to access low energy photons), large exciton radius (to permit use of larger particles thereby ameliorating surface effects), and nearly equal electron and hole effective masses (for high efficiency inverse Auger effect) [5].

Although efficient multiexciton generation is important, it is not useful if the excited carriers cannot be extracted or converted into useful photons. The lifetime and radiative recombination efficiency of excitons in $\mathrm{PbS}$ is an important probe of carrier recombination pathways. The decay lifetime for excitons has been observed to be unusually long in similar $\mathrm{PbS}$ particles $[1,6]$ and the presence of a "dark exciton" state has been recently proposed [7]. The term "dark exciton" is used to refer to an intrinsic quantum dot state that is optically forbidden [8]. If a dark exciton state lies at lower energy than the allowed HOMO-LUMO transition, the decay lifetime for an excited exciton can be extended by orders of magnitude. To address the question of the nature of the lowest excited state, we report here on the temperature dependence of the photoluminescence and photoinduced bleaching magnitudes and decay lifetimes.

", Mat. Res. Soc. Symp. Proc., vol. 974E, pp0974-CC06-01 ff. 


\section{THEORETICAL BACKGROUND}

The intensity of steady state HOMO-LUMO photoluminescence depends only on the occupation and radiative lifetime $\tau_{r}$ of the allowed HOMO -LUMO transition:

$$
I_{P L} \propto N_{L} \tau_{r}^{-1}
$$

where $N_{L}$ is the number of dots with an exciton in the HOMO-LUMO state. (We use the term "HOMO-LUMO exciton state" to indicate a state with an electron in the LUMO state and a hole in the HOMO state.) The occupation of the HOMO-LUMO exciton state can be measured directly using the steady state photoinduced bleaching magnitude of the HOMO-LUMO transition: $\Delta I_{P M} \propto N_{L}+N_{T}$. In the absence of traps, the ratio of PL to PM magnitude should yield the (temperature independent) radiative lifetime.

$$
\tau_{r}(T) \propto \frac{\Delta I_{P M}(T)}{I_{P L}(T)} .
$$

The number of dots $N_{L}$ with HOMO-LUMO excitons depends on the generation rate $G$, the excitation lifetime $\tau$, and the fraction of the lifetime that an exciton spends in the HOMOLUMO state. If there is a trap or dark exciton below the HOMO-LUMO state, then the exciton may spend only a small fraction of its life in the HOMO-LUMO state. The corresponding decay time depends on the temperature dependent ratio of occupations and the two lifetimes:

$$
\frac{1}{\tau_{\text {decay }}}=\frac{g_{L} e^{-\Delta E_{L T} / k T} / \tau_{L}+g_{T} / \tau_{T}}{g_{L} e^{-\Delta E_{L T} / k T}+g_{T}}
$$

where $g_{i}$ is the degeneracy of the $i$ th state and $E_{L T}$ is the energy difference between the two states.

Fundamental considerations of the HOMO-LUMO excitation cross-section predict a single exciton radiative lifetime of $\sim 400 \mathrm{~ns}$ [7] for $\mathrm{PbS}$ particles embedded in glass with HOMOLUMO transition at $0.8 \mathrm{eV}$ ( $3 \mathrm{~nm}$ radius). This lifetime should be relatively independent of temperature until thermally activated nonradiative recombination becomes important at high temperature. Experimental reports of the decay lifetimes for $\mathrm{PbS}$ in glass are more than ten times larger than the theoretical prediction, suggesting the presence of a trap or dark exciton state.

In one model for the dark exciton state, the energy difference between dark and allowed states is due to singlet-triplet splitting [7]. If this is the case, then the splitting should be small (of order 1-10 meV) and relatively insensitive to small variations in HOMO-LUMO energy.

\section{EXPERIMENT}

$\mathrm{PbS}$ nanoparticles were grown in glass from quenched $\mathrm{PbS}$-doped borosilicate samples by heat-treating at the temperature range from 540 to $580^{\circ} \mathrm{C}$ for 12 to 36 hours [6, 9-11]. The samples for this study were chosen to have a HOMO-LUMO peak near $0.8 \mathrm{eV}$; this corresponds to a mean particle radius of approximately $3 \mathrm{~nm}$. The absorption spectrum for representative glasses is shown in Fig. 1.

", Mat. Res. Soc. Symp. Proc., vol. 974E, pp0974-CC06-01 ff. 
Time resolved and cw photoluminescence and pump-probe measurements were carried out for sample temperatures from $77 \mathrm{~K}$ to $500 \mathrm{~K}$. The pumpprobe system is described in detail elsewhere[1]. A dye laser pumped by a nitrogen laser is used as the pump source for both luminescence and pump-probe kinetics. Laser pulses have a wavelength of $640 \mathrm{~nm}$ $(1.94 \mathrm{eV})$, duration of $\sim 5 \mathrm{~ns}$, and repetition rate of $\sim 10 \mathrm{~Hz}$. Typical excitation intensity of pump applied to the sample is

$<40 \mu \mathrm{J} / \mathrm{cm}^{2}$. The probe for time-resolved pump-probe studies is a $1550 \mathrm{~nm} \mathrm{cw}$ semiconductor laser. The response time for the Ge photodiode detector for this study is $1.0 \mu \mathrm{s}$. Time-resolved luminescence was excited with the same dye laser and detected with the same Ge photodiode detector and electronics.

Steady state luminescence and photomodulated transmission were excited with a $650 \mathrm{~nm}$ diode laser, chopped at 1000 $\mathrm{Hz}$, and detected with an uncooled $\mathrm{Ge}$ photodiode and lock-in amplifier. An HP $462 \mathrm{~A}$ voltage amplifier acted as a transimpedance amplifier for both oscilloscope and lock-in detection.

\section{RESULTS}

The intensity of the steady state photoluminescence (PL) and photoinduced bleaching (PM) is plotted against temperature in Figure 2. The intensity drops dramatically above room temperature, consistent with a thermally activated nonradiative recombination pathway. The intensity is only weakly dependent on temperature for lower temperatures, dropping slightly with decreasing $\mathrm{T}$, consistent with literature [7]. The difference in the PL and PM temperature dependence indicates the presence of trapping states.

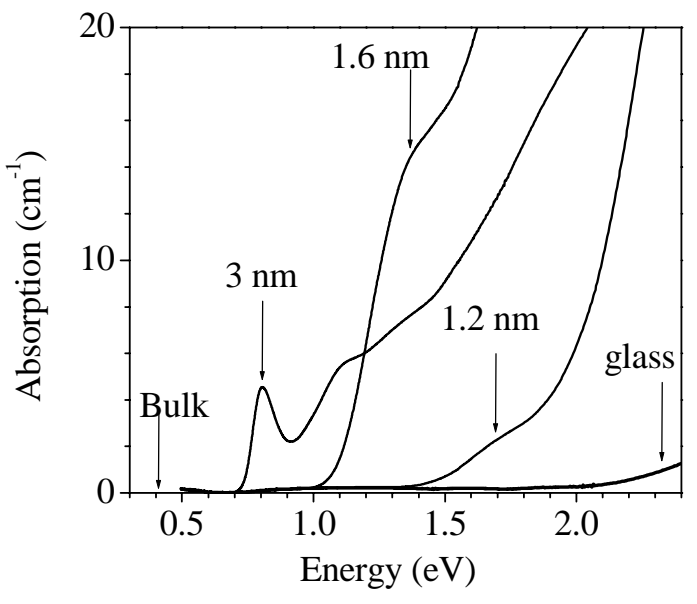

Fig. 1 - Optical absorption coefficient for a series of glasses in which $\mathrm{PbS}$ nanoparticles have been grown. The arrows denote the sizes that correspond to a lowest absorption peak at that energy. (from ref. [1]

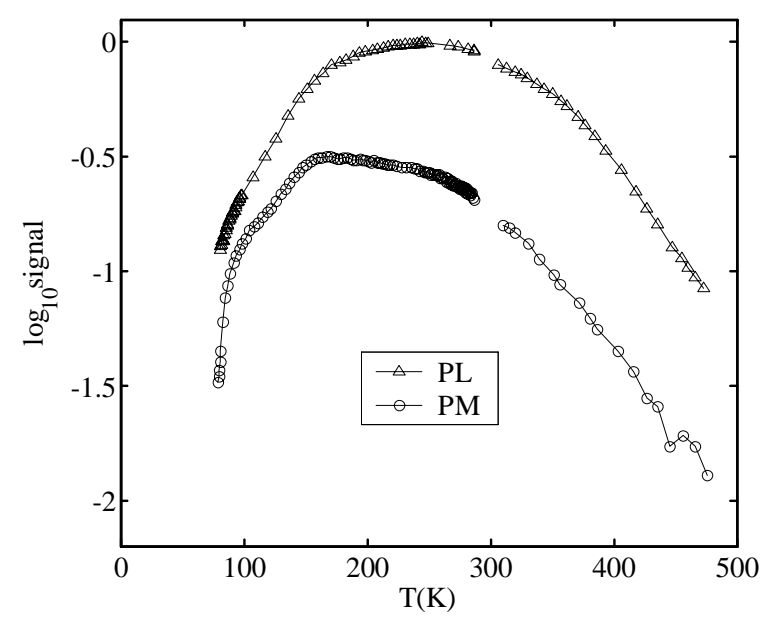

Figure 2 - Photoluminescence and photomodulation magnitude plotted against temperature. 


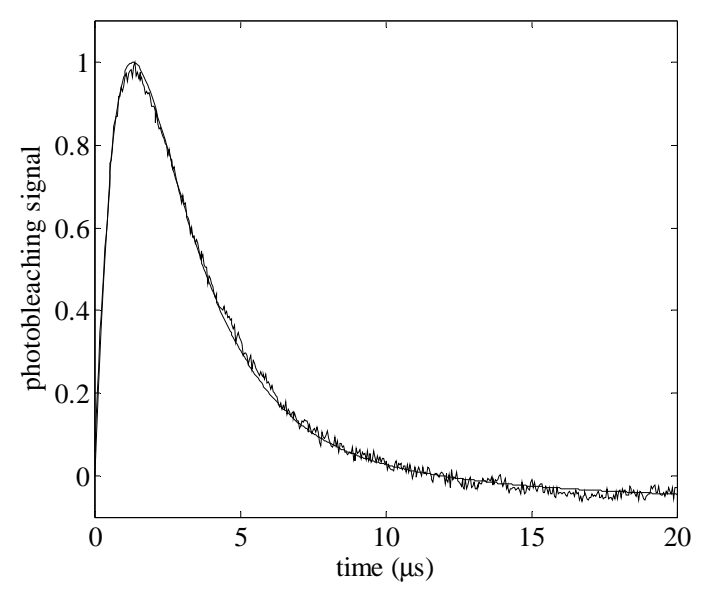

Figure 3 - Photoinduced bleaching signal at $1550 \mathrm{~nm}$ at $295 \mathrm{~K}$. The solid line is a fit using a single exponential decay time of $2.0 \mu \mathrm{s}$. Response time of the Ge detector is $1.0 \mu \mathrm{s}$.

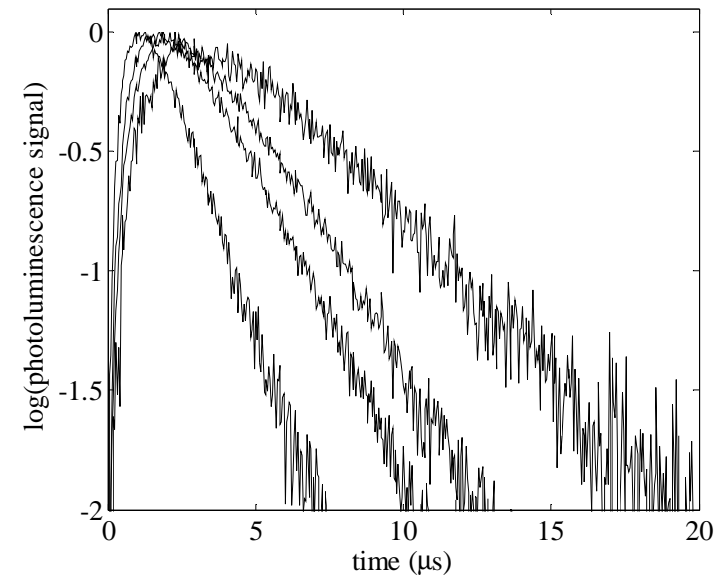

Figure 4 - Photoluminescence decay intensity plotted against time for several temperatures. Long lifetime curve $\sim 100 \mathrm{~K}$, short lifetime $\sim 400 \mathrm{~K}$

An example of the time dependence of photoinduced bleaching is shown in Figure 3. The signal rise time is determined by the detector response time of $1 \mu$ s. The decay time is due to convolution of detector and sample response and is consistent with a sample decay time of 2.0 $\mu$ s. The solid fit curve is the result of the convolution of exponential detector response and exponential sample response.

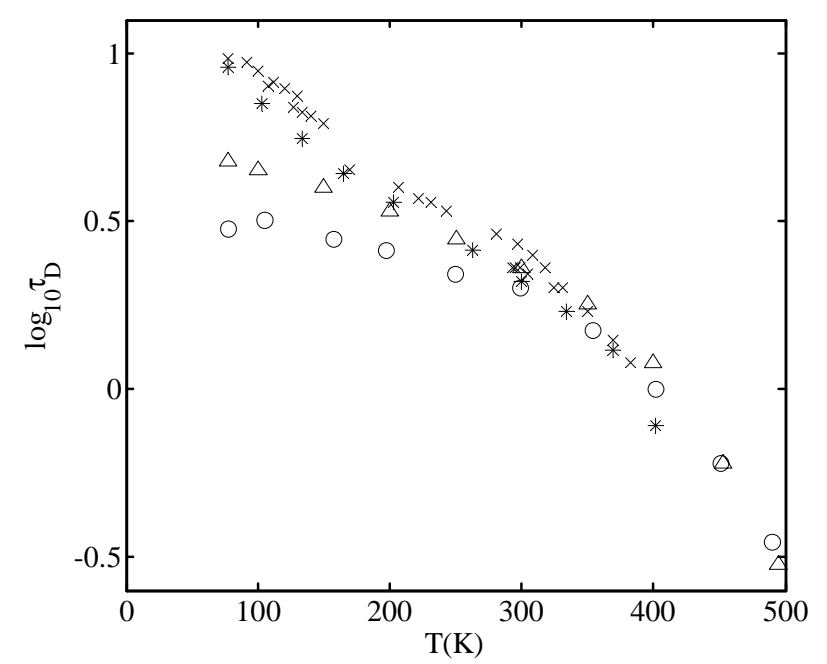

Figure 5 - Decay lifetime plotted against temperature for several locations on the sample. Each symbol denotes a different location.
The time dependence of photoluminescence is shown for several temperatures in Figure 4. The PL decay time increases monotonically with decreasing temperature as shown in Figure 5. Most notable is the fact that the decay time depends on the location on the sample. In all locations the spectrum is the same, but the lifetime at low temperature changes by as much as a factor of 5 . 


\section{CONCLUSIONS}

Steady state photoluminescence is consistent with earlier reports and with a dark exciton interpretation, but comparison to the photomodulated bleaching signal suggests that traps may play an important role. The fact that the decay lifetime for both PL and PM depends on position on the sample is further evidence of a defect or trap, rather than a dark exciton.

\section{ACKNOWLEDGEMENTS}

We thank Pratima Rao for supplying PbS doped glass for nanoparticle growth and Robert Doremus for helpful discussions on nanoparticle growth. Nicholas Scaptura performed preliminary temperature-dependent steady state PL and PM measurements. Alex Pickle performed photoluminescence spectral measurements.

\section{REFERENCES}

1. F. Huang, A. Filin, R. Doremus, P.N. Rao, and P.D. Persans, Long-Lifetime Nonlinear Absorption of Pbs Quantum Dots. Mat. Res. Soc. Symp. Proc., 737, (2003) 163.

2. R.D. Schaller and V.I. Klimov, High Efficiency Carrier Multiplication in Pbse Nanocrystals: Implications for Solar Energy Conversion. Phys. Rev. Lett., 92, (2004) 186601.

3. R.J. Ellingson, M.C. Beard, J.C. Johnson, P. Yu, A.I. Micic, A.J. Nozik, A. Shabaev, and A.L. Efros, Highly Efficient Multiple Exciton Generation in Colloidal Pbse and Pbs Quantum Dots. Nanoletters, 5, (2005) 865.

4. $\quad$ A.J. Nozik, Quantum Dot Solar Cells. Physica E-Low-Dimensional Systems \& Nanostructures, 14, (2002) 115-120.

5. M. Califano, A. Zunger, and A. Franceschetti, Efficient Inverse Auger Recombination at Threshold in Cdse Nanocrystals. Nano Letters, 4, (2004) 525-531.

6. P.D. Persans, A. Filin, F. Huang, A. Vitek, P.G.N. Rao, and R.H. Doremus. Trap Effects in Pbs Quantum Dots. in Quantum Dots, Nanoparticles and Nanowires Symposium, 1-5 Dec. 2003. 2004. Boston, MA, USA: Mater. Res. Soc.

7. R. Espiau de Lamaestre, H. Bernas, D. Pacifici, G. Franzo, and F. Prioli, Evidence for a "Dark Exciton" State of Pbs Nanocrystals in a Silicate Glass. Appl. Phys. Lett., 88, (2006) 181115-2.

8. M. Nirmal, D.J. Norris, M. Kuno, M.G. Bawendi, A.L. Efros, and M. Rosen, Observation of Dark Exciton in Cdse Quantum Dots. Phys. Rev. Lett., 75, (1995) 3728.

9. P.N. Rao, Cadmium Sulfide and Lead Sulfide Quantum Dots in Glass: Processing, Growth, and Optical Absorption, in Materials Science, Rensselaer Polytechnic Institute: Troy, (2001).

10. F. Huang, Nonlinear Optical Studies of the Properties of Nanoparticles, in Physics, Rensselaer Polytechnic Institute: Troy, (2003) 122.

11. F. Huang, A. Filin, R. Doremus, P. Rao, and P.D. Persans, Long-Lifetime Nonlinear Absorption of Pbs Quantum Dots. Mat. Res. Soc. Symp. Proc., 737, (2003) 163.

", Mat. Res. Soc. Symp. Proc., vol. 974E, pp0974-CC06-01 ff. 